\title{
DIVISION ALGEBRAS OF DEGREE 4 AND 8 WITH INVOLUTION
}

\author{
BY S. A. AMITSUR, L. H. ROWEN 1 , AND J. P. TIGNOL 2
}

ABSTRACT. Examples are given of division algebras with involution $(*)$ of the first kind, one of degree 8 which is not a tensor product of quaternion subalgebras, the other of degree 4 which is not a tensor product of (*)-invariant quaternion subalgebras.

Suppose $D$ is a division algebra with center $F$, and $[D: F]<\infty$. Then $[D: F]$ $=n^{2}$ for suitable $n ; n$ is the degree of $D$, and $D$ is a quaternion $F$-algebra when $\operatorname{deg}(D)=2$. We further assume $D$ has characteristic $\neq 2$, and has an involution (*) of the first kind, i.e. (*) is an anti-automorphism of degree 2 which fixes $F$. This situation is treated in depth in [1, Chapter 10], and it arises if and only if $D$ has exponent 2 in the Brauer group, i.e. $D \otimes D^{\mathrm{op}} \approx M_{n_{2}}(F)$, the algebra of $n^{2} \times n^{2}$ matrices over $F$. Thus, in this case, the degree of $D$ is a power of 2 . Until now, the only known such algebras were tensor products of quaternion $F$-subalgebras.

QUESTION 1. Is $D$ necessarily a tensor product of quaternion $F$-subalgebras?

QUESTION 2. Is $D$ necessarily a tensor product of (*)-invariant quaternion $F$-subaligebras?

Question 1 dates back about 60 years; Albert [1] showed it is true when $\operatorname{deg}(D) \leqslant 4$. The main object of this paper is to give a counterexample for degree 8. Also, we shall give a counterexample to Question 2 for degree 4, which is clearly sharp. (Incidentally, for symplectic involutions, question 2 has no counterexample of degree 4, cf. [3, Theorem B] .) Our counterexample makes the following result of Tignol [4] sharp: If $\operatorname{deg}(D)=8$ then $M_{2}(D)$ is a tensor product of quaternion subalgebras. A more detailed description of our methods will appear in the Israel Journal of Mathematics.

The main idea is to use the generic abelian crossed products of [2], modified slightly to account for the presence of an involution. Suppose $R$ is an abelian crossed product, i.e. $D$ has a maximal subfield $K$ Galois over $F$, having Galois group $G=\left\langle\sigma_{1}\right\rangle \oplus \cdots \oplus\left\langle\sigma_{q}\right\rangle$, a direct sum of cyclic groups, and for our purposes we assume that $\sigma_{i}$ has order 2 . Then, choosing $z_{i}$ such that $\sigma_{i}(x)=$ $z_{i} x z_{i}^{-1}$ for all $x$ in $K$, we define $u_{i j}=z_{i} z_{j} z_{i}^{-1} z_{j}^{-1}$ and $b_{i}=z_{i}^{2}$, elements of $K$. [2, Lemma 1.2] gives the following conditions for all $i$ (where $N_{i}(x)=x \sigma_{i}(x)$ by definition).

Received by the editors November 16, 1978.

AMS (MOS) subject classifications (1970). Primary 16A40, 16A28; Secondary 15 A66.

1 Research of the second author is supported by the Anshel Pfeffer Chair.

2 The third author is grateful to Professor J. Tits for enlightening conversations. 
(1) $u_{i i}=1$ and $u_{i j}^{-1}=u_{j i}$ for all $i$;

(2) $\sigma_{i}\left(u_{j k}\right) \sigma_{j}\left(u_{k i}\right) \sigma_{k}\left(u_{i j}\right)=u_{i j} u_{j k} u_{k i}$ for all $i, j, k$;

(3) $N_{i}\left(N_{j}\left(u_{i j}\right)\right)=1$ for all $i, j$;

(4) $\sigma_{j}\left(b_{i}\right) b_{i}^{-1}=N_{i}\left(u_{j i}\right)$ for all $i, j$.

Conversely, these conditions for given elements of a Galois field extension $K$ of $F$ with abelian Galois group $G$, define a simple $F$-algebra $R$ of dimension $G^{2}$ and center $F$.

THEOREM 1. Notation as above, $R$ has an involution iff, modifying the $u_{i j}$ and $b_{i}$ suitably, we can satisfy (1)-(4) above, as well as the following extra conditions, where $\tau \in G$ is arbitrarily chosen:

(5) $\tau\left(u_{i j}\right) \sigma_{i} \sigma_{j}\left(u_{i j}\right)=1$ for all $i, j$,

(6) $\tau\left(b_{i}\right)=b_{i}$ for all $i$.

Proof. $(\Rightarrow)$ Using the proof of [3, Propositions 5.4 and 5.5], one sees easily that $R$ has an involution iff $R$ has some involution (*) whose restriction to $K$ is $\tau$. For all elements $k$ in $K, z_{i} k=\sigma_{i}(k) z_{i}$, taking (*) on both sides, and substituting, shows $z_{i}^{*} \in z_{i} K$, so we can replace $z_{i}$ by $z_{i} \pm z_{i}^{*}$, (5) and (6) follow easily.

$\Leftrightarrow$ Define (*) by $\left(\Sigma k_{\alpha} z_{1}^{\alpha} \cdots z_{q}^{\alpha} q\right) *=\Sigma z_{q}^{\alpha} q \cdots z_{1}^{\alpha}{ }_{1} \tau\left(k_{\alpha}\right), k_{\alpha}$ in $K$, using (5) and (6) to prove (*) is an involution. Q.E.D.

Write $U$ for $\left\{u_{i j} \mid 1 \leqslant i, j \leqslant q\right\}$ and $B$ for $\left\{b_{i} \mid 1 \leqslant i \leqslant 3\right\}$. We restrict ourseves to the case $q=3$, i.e. $K$ is a given Galois extension of $F$ with Galois group $\mathbf{Z}_{2} \oplus \mathbf{Z}_{2} \oplus \mathbf{Z}_{2}$. Thus, we can write $K=F\left(\xi_{1}, \xi_{2}, \xi_{3}\right)$ with $\xi_{i}^{2} \in F, \sigma_{i}\left(\xi_{i}\right)=$ $-\xi_{i}$, and $\sigma_{i}\left(\xi_{j}\right)=\xi_{j}$ for $j \neq i$. Also take $\tau=\sigma_{1} \sigma_{2} \sigma_{3}$.

THEOREM 2. Given B, we can find $U$ satisfying (1)-(6) above iff there are elements $v_{1}, v_{2}, v_{3}$ in $K$ satisfying

(2) $v_{1} v_{2} v_{3}=1$,

(3) $N_{i}\left(v_{i}\right)=1$,

as well as the following conditions for every permutation $\pi$ of $(1,2,3)$ :

(4) $\sigma_{\pi 1}\left(b_{\pi 2}\right) b_{\pi 2}^{-1}=\left(N_{\pi 2}\left(v_{\pi 3}\right)\right)^{s g \pi}$;

(5)' $b_{\pi 1} \in F\left(\xi_{\pi 2} \xi_{\pi 3}\right)$.

PRoof. Straightforward computations, defining $v_{1}=u_{23}=u_{32}^{-1}, v_{2}=$ $u_{31}=u_{13}^{-1}, v_{3}=u_{12}=u_{21}^{-1}$, and $u_{11}=u_{22}=u_{33}=1$. Q.E.D.

The proof of Theorem 2 also shows (1)-(5) imply (6).

THEOREM 3. Given $v_{1}, v_{2}, v_{3}$ satisfying $(2)^{\prime},(3)^{\prime}$, there exists $B$ satisfying (4)' and (5)' such that, for every permutation $\pi_{\text {, }}$

$$
b_{\pi 1}=F\left(\xi_{\pi 2} \xi_{\pi 3}\right) \cap F\left(\xi_{\pi 2}\right) N_{\pi 1}(K) \cap F\left(\xi_{\pi 3}\right) N_{\pi 1}(K) .
$$

Proof. Define $u_{i j}$ as in Theorem 2, so that (1), (2), and (3) are satisfied. By [2, equation (14)], which should read $a_{k} \sigma_{i}\left(a_{k}^{-1}\right)$ etc., we obtain the elements 
$a_{i}$, which we rename $b_{i}$, satisfying (4). We readily get $(2)^{\prime}-(5)^{\prime}$. By Hilbert's theorem 90, we have $y_{i}$ such that $v_{i}=\sigma_{i}\left(y_{i}\right) y_{i}^{-1} ; b_{1} N_{1}\left(y_{3}\right)$ is fixed under $\sigma_{3}$, so $b_{1} N_{1}\left(y_{3}\right) \in F\left(\xi_{2}\right)$ and $b_{1} \in F\left(\xi_{2}\right) N_{1}\left(y_{3}^{-1}\right)$. Likewise $b_{1} N_{1}\left(y_{2}^{-1}\right) \in F\left(\xi_{3}\right)$ etc. Q.E.D.

Suppose now we are given $b \in K$ satisfying

(7) $b \in F\left(\xi_{2} \xi_{3}\right) \cap F\left(\xi_{2}\right) N_{1}(K) \cap F\left(\xi_{3}\right) N_{1}(K)$. Then, taking $b=a_{2} N_{1}(w)=a_{3} N_{1}\left(w^{\prime}\right)$, put $v_{2}=w^{-1} \sigma_{2}(w), v_{3}=\left(w^{\prime}\right)^{-1} \sigma_{3}\left(w^{\prime}\right)$ and $v_{1}=\left(v_{2} v_{3}\right)^{-1}$. Theorems 3 and 2 then apply, giving $B$ and $U ; b \in F b_{1}$, so we replace $b_{1}$ by $b$. Form the corresponding abelian crossed product $R$. The generic abelian crossed product $R^{\prime}$ (cf. [2]) is a division ring with involution, by Theorem 1. If $R^{\prime}$ is a tensor product of quaternion subalgebras, then $R^{\prime}$ has some set of square-central elements $r_{1}^{\prime}, \ldots, r_{64}^{\prime}$, independent over $\operatorname{Cent}\left(R^{\prime}\right)$ with $r_{i}^{\prime} r_{j}^{\prime}= \pm r_{j}^{\prime} r_{i}^{\prime}$ for all $i, j$. An argument based on taking leading monomials (cf. [2, Lemma 2.1]) then shows there is such a set of elements of $R$, each having the form $k_{i} z_{1}^{i} z_{2}^{i_{2}} z_{3}^{i_{3}}$, with $k_{i} \in K$. In particular, one of these elements must be of the form $k z_{1}$, implying some $\alpha=\left(k z_{1}\right)^{2}=b N_{1}(k)$, so $b \in F N_{1}(K)$. Thus, to answer Question 1 negatively, we need to find $F, K=F\left(\xi_{1}, \xi_{2}, \xi_{3}\right)$, and $b \in K$, such that (7) holds and $b \notin F N_{1}(K)$. (The counterexample will be $R^{\prime}$.)

Take $F=Q(\lambda)$, the field of rational functions in one indeterminate $\lambda$ and $\xi_{i}$ such that $\xi_{1}^{2}=-1, \xi_{2}^{2}=-\left(\lambda^{2}+1\right)$, and $\xi_{3}^{2}=\lambda$, with $b=\xi_{2} \xi_{3}$. Then (7) holds. If $b \notin F N(K)$ then $\lambda\left(\lambda^{2}+1\right) \in N_{1}\left(F\left(\xi_{1}\right)\right)\left(N\left(F\left(\xi_{1} \xi_{2}\right) / F\right) \cap N\left(F\left(\xi_{2} \xi_{3}\right) / F\right)\right)$, where $N($ ) denotes the norm of a field extension. (This step is not easy.) This is impossible, seen by taking polynomials modulo 2 .

Similarly, Question 2 has a counterexample iff there is a field extension $K=F\left(\xi_{1}, \xi_{2}\right)$ of $F$ and some $b \in F N_{1}(K)$, with $b \notin F k^{2}$ for all $k$ in $F\left(\xi_{2}\right)$. Take $F=Q(\lambda), \xi_{1}^{2}=2, \xi_{2}^{2}=\lambda$, and $b=\lambda-1+2 \xi_{2}$.

\section{REFERENCES}

1. A. A. Albert, Structure of algebras, Amer. Math. Soc. Colloq. Publ. no. 24, Amer. Math. Soc., Providence, R.I., 1961.

2. S. A. Amitsur and D. Saltman, Generic abelian crossed products, J. Algebra 51 (1978), 76-87.

3. L. Rowen, Central simple algebras, Israel J. Math. 29 (1978), 285-301.

4. J. Tignol, Sur les classes de similitude de corps à involution de degré $8, \mathrm{C}$. R. Acad. Sci. Paris, Sér A 286 (1978), 875-876.

5. - Décomposition et descente de produits tensoriels d'algebres de quaternions, Rap. Sém. Math. Puré UCL 76 (1978).

DEPARTMENT OF MATHEMATICS, HEBREW UNIVERSITY OF JERUSALEM, JER USALEM, ISRAEL ISRAEL

DEPARTMENT OF MATHEMATICS, BAR ILAN UNIVERSITY, RAMAT GAN,

DEPARTMENT OF MATHEMATICS, CATHOLIC UNIVERSITY OF LOUVAIN, LOUVAIN-LA-NEUVE, BELGIUM 